\title{
'||||||||||||||||||||||||||||||||||||||||||||||||||||||||||||||||||||.
}

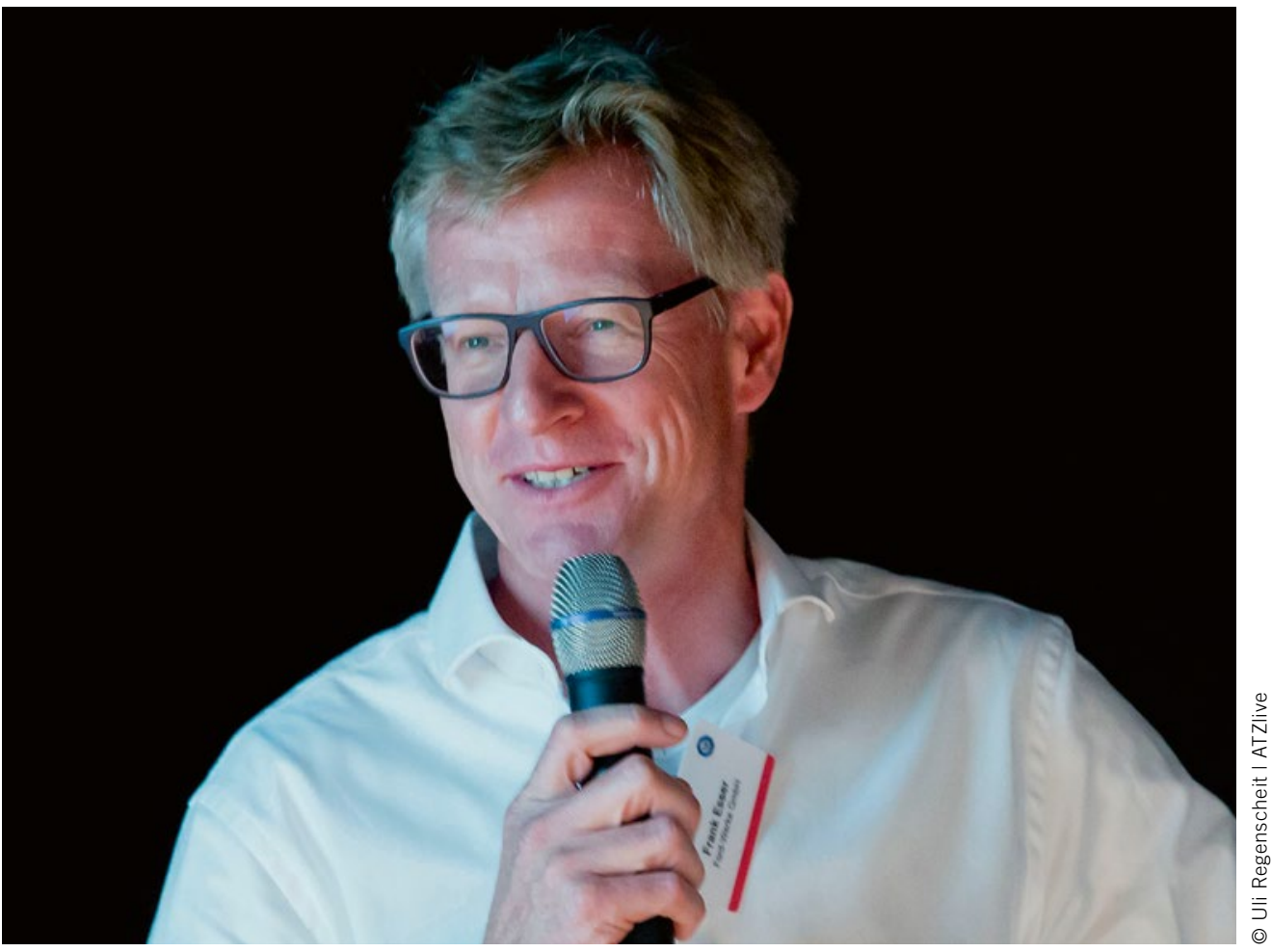

Dipl.-Ing. Frank Esser Supervisor

Advanced Driving Attribute

Methods \& Tools,

Chassis and Vehicle Architecture

\& Technology,

Ford Research \& Advanced

Engineering,

ATZ Scientific Advisory Board

\section{Few Prototypes - Good Driving Dynamics}

The approach is not new to any of us - after all, the driving dynamics development departments have long been asked to reduce the number of physical prototypes. The application effort is also multiplied by the newly emerging electrified drive versions, especially as regards the design of the active chassis elements and assistance functions.

What can a new path in chassis and driving dynamics development look like? Can the interaction between ADAS and classic driving dynamics work? All companies are working intensively on getting away from the extremely expensive full vehicle prototype in the early construction phase and moving both the functional verification and the basic tuning to the laboratory or the CAE world. The following must be fulfilled: The component and system models must be sufficiently validated, the interfaces clearly defined, and the functional goals precisely described.

This essentially requires early and intensive collaboration between the car makers and their suppliers. This includes early exchange about the tool chains, expectations of the quality of the models, the necessary validation and common definition of the interfaces.

And how can this support the detail optimization done by us experts? In my view, the basis is in the common work on the models. This should start with the model of the component, continue through the system and end in the full vehicle simulation. It is important to be clear together about the following: Is a model that only reflects the properties of the component sufficient, or do I need a physically based model that enables me to optimize the design of the component?

These and other questions can only be resolved jointly by the OEM and the supplier. To do this, the OEM needs a high level of expertise in the area of virtual development, but also the associated model parameterization and necessary physical tests. However, a competent supplier in this area is just as important. An important step for efficiently implementing virtual development on the OEM and supplier side is the creation of appropriate structures and a defined integration into the development process. Just as clear phases are defined for prototype construction, so it must be for the virtual process.

With today's complexity of the variants, it is hardly possible to physically test all variants and even more so to optimize them for robustness. The time and parts for it are not available. This is where the well-validated model comes in, with which the full vehicle properties can be tested and optimized. To do this, the model must be well understood, you need to know for which area it is validated, where you can rely on the model. We see great potential in shifting the final coordination to prototypes from pre-series production. 